\title{
An Intelligent Model for Road Traffic Management in the Motorway Network Around Barcelona
}

\author{
J. Cuena, J. Hernández, M. Molina \\ Department of Artificial Intelligence \\ Technical University of Madrid \\ Campus de Montegancedo s/ $n$, Boadilla del Monte 28660, Spain \\ Phone: (34-1) 352-48-03, Fax: (34-1) 352-48-19 \\ E-mail: \{jcuena,phernan,mmolina\}@dia.fi.upm.es
}

\begin{abstract}
This paper presents an advanced knowledge-based environment to develop real time traffic management applications called TRYS. The building process supported by the architecture is guided by the progressive definition of knowledge features from the knowledge level to the symbolic level. Firstly, the problem is presented showing the shortcomings perceived in the state of the art of traffic management systems. Secondly, a description of the KSM tool, aimed at supporting the organization of structured models at the knowledge level is commented. Thirdly, the generic model, intended to deal with traffic management, is described using the KSM format. Finally, the domain model of the application developed for Barcelona is described.
\end{abstract}

Keywords

Knowledge-based systems, adaptive traffic management, knowledge architectures

\section{INTRODUCTION}

Traffic management systems must be reactive to the different states of traffic flow in a controlled network (a network equipped by sensors and data communication facilities allowing to get real time data in a central computer and to diffuse signals from this central computer). These systems evolved from an initial approach based on a library of signal plans which were applied on a time based pattern, to an intelligence for understanding traffic situations in real time integrating a model for decision making (Bretherton et al. 1981), (Mauro, 1989). Nevertheless, the experience in using such systems showed deficiencies when the traffic situation became specially problematic and the intervention of the operator was necessary and almost customary in most installations.

The above considerations suggested a need to complement the existing traffic control systems (including pre-calculated plan systems, dynamic systems and VMS systems) with an additional layer where the strategic knowledge, currently applied by human operators, may be applied to understand the specific processes of congestion development, and corresponding 
actions for alleviating the problem may be modeled. From this viewpoint, the technology of knowledge-based systems was considered adequate for designing and implementing suitable knowledge structures to formulate conceptual models for traffic analysis and management.

\section{THE KNOWLEDGE STRUCTURING TOOL}

The KBS structuring and the identification of knowledge level forms made feasible the existence of a new concept of application development tools mainly based in the translation from assumed structures at the knowledge level to structured symbolic models. The KMS tool (Cuena, Molina, 1994) presented is an evolved environment of previous proposals (Bholz et al. 1991), (Musen et al. 1993), (McIntryre, 1993), using as structuring concept the knowledge unit.

A knowledge unit does not follow the classical computational division of process and data. On the contrary, it models a knowledge body to be identified in the modeling process of the intelligence of an agent. Internally, the knowledge unit defines (see Figure 1) not only what tasks the unit performs, which provides a functional description, but also what the unit knows, required to perform these tasks, which offers a cognitive description formulated by a group of component knowledge areas. These knowledge areas are capable of performing basic tasks used as elementary inference steps by the methods fulfilling the functionalities of the knowledgeunit.

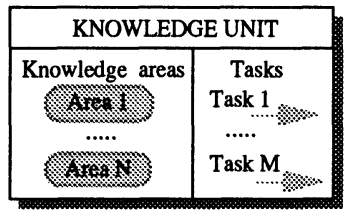

Figure 1 Format of a knowledge unit.

A general description of a knowledge model in KSM integrates three different views:

- Knowledge area view: a graph hierarchy of knowledge units related by the property is used by .

- Task view: several trees of tasks implicit in the knowledge units structure. Each task is carried out by a method.

- Vocabulary view: several conceptual vocabularies attached to primary knowledge units, to share common concepts among different knowledge bases. Their content are attribute-value domain couples that can be assigned to different concepts as in (Brachman et al. 1985).

$\mathrm{KSM}$, then, contributes to knowledge modularity by proposing the synthetic knowledge area view. This view is more global than the task view because the knowledge perspective generally implies several task tree views given that a unit may offer several functionalities.

KSM may support the development of a knowledge oriented design method through several steps of progressive evolution from conceptual abstractions to computable units:

1) Knowledge level description of the model, where the knowledge view structure is designed implying the different functionalities through trees of tasks.

2) Generic symbol level design, where design decisions are taken on the conceptual vocabularies, the knowledge bases of the primary units and the inference methods.

3) Domain symbol level design, where knowledge bases for specific declarative components, designed to deal with a class of problems, are introduced in the structure defined in 2).

4) Validation and refinement loop.

5) Final installation and maintenance. 


\section{THE TRYS MODEL}

The TRYS system is a knowledge representation environment supporting a KSM generic symbol level model to perform traffic management at a strategic level in urban, interurban or mixed areas. The traffic network to be supervised is divided in several sections called problem areas. A problem area is a part of a network where frecuent problems appear and where a local traffic control is performed. The conclusions obtained from the local analysis are consistently integrated using strategical criteria from a global perspective, i.e. applying the knowledge of a coordination intelligence.

The cognitive and traffic models presented below were in depth presented in (Cuena et al. $1995,1996)$, so the attention is focused on their application to the traffic problem in Barcelona.

\subsection{The knowledge level}

The knowledge view of the TRYS system consist of a hierarchy of knowledge units obtained integrating the following elements:

- A top level unit with two knowledge areas: local control and coordination.

- A collection of local control areas every one described by a knowledge units structure in the way shown in Figure 2.

- A coordination area described by a knowledge units structure in the way shown in Figure 3.

The goal of every local control unit is double: (i) detect and diagnose the traffic problems present within the local area and, (ii) propose control actions using the available signal devices to improve the traffic conditions. The knowledge areas supporting this activity are two (see Figure 2):

- The Traffic Problems knowledge unit which provides information about the present and short term predictable congestions together with associated explanations about their causes.

- The Traffic Control knowledge unit which elaborates signal proposals in terms of VMS messages for the incoming traffic and/or level of containment (low, medium, high, total) for the traffic lights in the controlled intersections or entrance ramps.

Both units share all the information concerning the physical structure of the local area which is provided by the Network knowledge unit.

The other unit supporting the Traffic Problems knowledge unit is the Problem Scenarios unit. It applies a classification method on a set of predefined problem scenarios representing classes of problems at different levels of detail. When some of these problems is selected (i.e. it is active) an explanation of its possible causes is elaborated. The information required to build the explanation is provided by the Network unit using a qualitative simulator of the traffic assignment.

The task performed by the Traffic Control knowledge unit can be decomposed in two parts:

- to look for proposals of VMS panel displays which may induce drivers to take paths that do not pass through congested areas.

- to modify the phases in the traffic lights to (i) retain the upstream flows in those paths with the greatest influence on the state of the critical sections and (ii) to improve the capacity downstream the critical sections.

The reasoning performed by this knowledge unit is based on a generate and test method supported by the knowledge units shown in Figure 2. First, the VMS Path Use knowledge unit looks for sets of messages that redistribute the traffic between every origin-destination couple in alternative paths. Then, the Traffic Lights Capacity unit searches for signal plans which reduce the upstream flows or increase the downstream capacity or both. Finally, both types of 
control proposals are consistently combined. To meet this goal the consistency between messages and phase variation is tested with the traffic assignment simulator of the Network unit which is used to evaluate the level of improvement in the excess value.

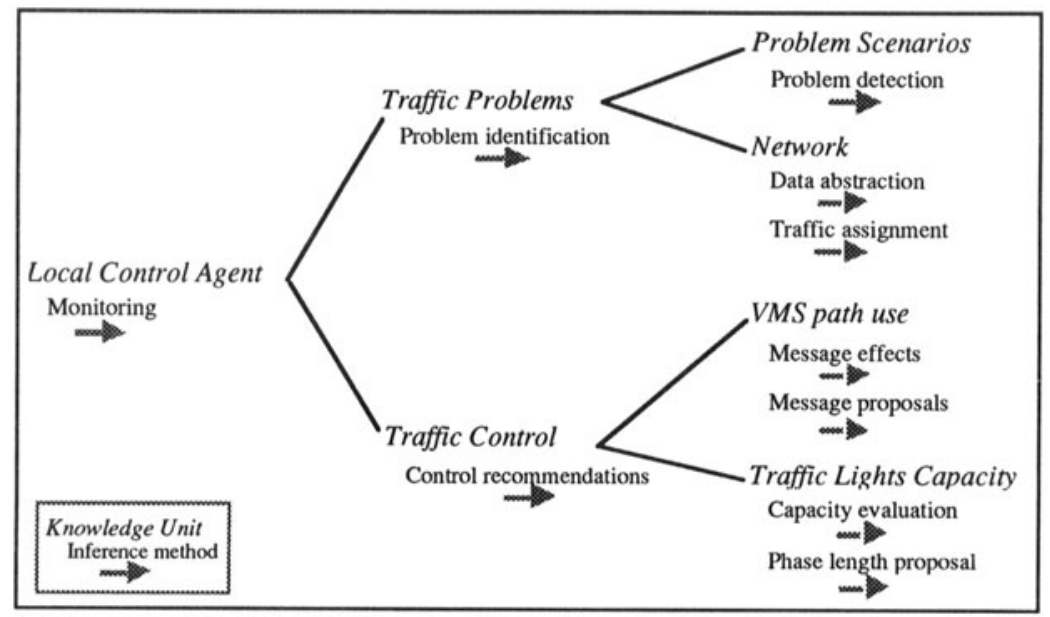

Figure 2 Knowledge view of the local control agent knowledge.

The VMS Path Use unit includes declarative knowledge relating control states with states of traffic behavior. The Traffic Lights Capacity unit makes use of a similar version of the previous knowledge to the case of the traffic lights control.

The result of the individual reasoning performed by a Local Control Agent is a set of control proposals for its problem area. All these proposals are analyzed by the Coordinator with the aim of building global proposals by coherently synthesizing the local proposals in signal recommendations for the whole network. This funtionality is performed through a propose and revise method supported by three knowledge units (see Figure 3):

- knowledge to evaluate the compatibility of the different proposals delivered by the local agents included in the Proposals Compatibility unit.

- knowledge to solve coordination conflicts within the Agent Priorities unit.

- knowledge to deduce complementary signals that improve the global impact of the previous proposals included in the Proposals Completion unit.

The knowledge used by the Proposals Compatibility unit is a set of characterizations of nogood situations of two types: (i) physical conflicts caused by different actions on the same signal device and, (ii) semantic incoherence between proposals which provoke undesirable effects on the traffic behavior. When some of this incompatibilities appear, the Agent Priorities unit make use of different control criteria (the importance of the area, the value of the state variables, etc) to decide which local agent must change its proposal. Sometimes, the control proposals generated in the previous steps can be completed with additional messages choosen from a global strategical view. This knowledge is organized in a collection of prototype situations relating features of internal signals and states with completion signals for the entries to the controlled areas. 


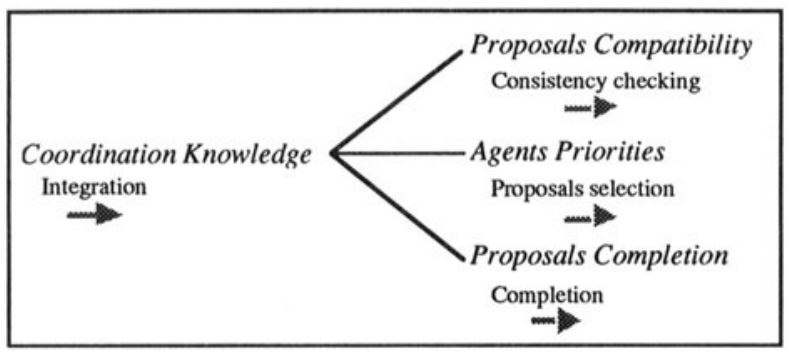

Figure 3 Knowledge view of the coordination knowledge agent.

\subsection{The generic symbol level}

To define a model at the generic symbol level the next steps have to be followed:

- define the conceptual vocabularies.

- decide the specific representation of the basic units.

- formulate the different reasoning methods for the high level units.

The conceptual vocabulary includes a hierarchy of the traffic concepts to be used and their characteristics. The specific values of these concepts are defined at the domain level associated to a particular application.

The Network unit is formulated by an ad hoc traffic network primitive supported by a declarative description of the network graph and with two methods that perform data abstraction of high level concepts and traffic volume assignment according to path use information.

The Problem Scenarios basic unit is formulated as a frame base of problem scenarios. This frames are defined with a collection of state variables characterizing the observed and short term traffic behavior as well as the signal state of the control area. This knowledge unit uses a reasoning method named 'match' which evaluates the matching degree of the real situation and the collection of problem scenario frames until a set with the best matching frames is generated.

The VMS Path Use primitive is also formulated as a frame base with a slot for every O-D couple. The value of these slots is the set of the main paths between $O$ and $D$ with an estimated percentage of its use. Besides, there are slots for the states of the VMS panels in such a way that for every scenario of messages a pattern of path uses is defined. This frame base can be used in two directions: to infer path uses from states of VMS panels or to infer configurations of messages to modify problematic path uses. In both cases, the same model for pattern matching is applied.

A rule base primitive is assigned to the Proposals Compatibility knowledge unit within the coordination model. These rules represent no-good situations and include in the left hand side a description of the conditions that make two control actions incompatible.

A set of priority tables, organized in different levels of request, is assigned to the Agent Priorities unit. The inference procedure tries to satisfy the first level for the maximum number of local agents. If this is not possible, because there are incompatible control actions, then the procedure tries to satisfy the next level and so on reducing the set of agents to be satisfied. It finishes when a level where no incompatibilities are detected is reached.

Finally, a frame primitive is assigned to the Proposals Completion knowledge unit. Every frame includes slots describing the current state of the control proposal (delivered by the consistence and priority units), slots describing the global state of the whole network and additional messages for unused VMS panels. 


\subsection{The Domain Model in Barcelona}

The proposed generic model allows an expert user on traffic management to define a specific domain model by fulfilling the declarative concepts used to formulate the knowledge about problem scenarios, network structure, coordination, etc. These knowledge bases can be inspected and improved by the traffic experts according to the experience gained with the dayly operation.

These criteria have been applied on an extensive network in Barcelona that includes the ring road of the city and all the main accesses. The problem in Barcelona was to develop a traffic management system that receives real time data and generates control plan proposals for congestion situations that may simultaneously appear in different parts of the network. These control proposals need to be supported with explanations to give the traffic operator confidence on taking the final decision.

The control requirements in the city determined the structure of the TRYS model with one coordinator and 18 local control agents. These number of agents was obtained considering both senses of traffic for every road and a partition of the ring road in four. The network areas assigned to the agents are control dependent and therefore some agents have to share control devices.

The traffic control infrastructure available includes 52 VMS panels, 3 traffic ligth controlled intersections, ramp metering in 7 entrances to the ring road, and more than 300 single and double loop detectors. These devices are connected with the Traffic Control Center through fiber optics communications which makes possible receiving data from sensors (every minute). These data are recorded by the Network unit and analyzed to deduce the signal information, the basic traffic data (i.e. speed, occupancy, flows) and the aggregated data (i.e. traffic volume generated in an entrance node, spatial gradient of speed) required by the other knowledge units in the system. The Traffic Problems unit compares the situation described by these data with those in the frame base of problem scenarios to identify if the current situation is free flow, dense traffic, incident congestion or overflowing congestion.

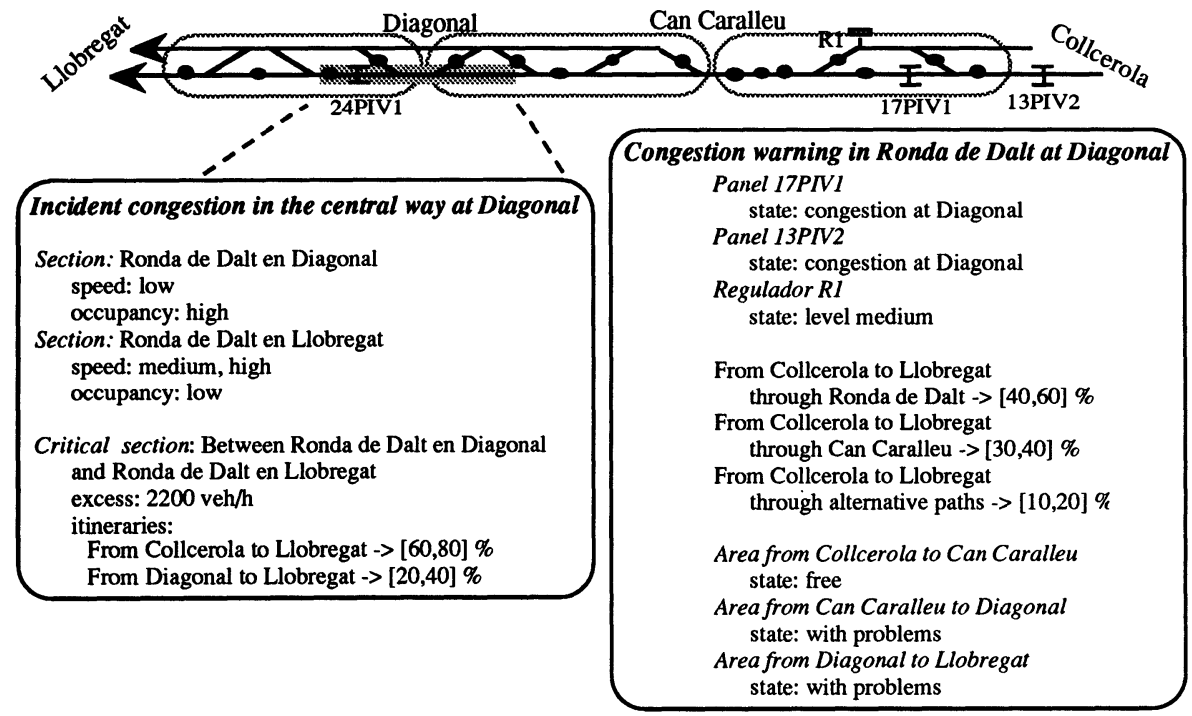

Figure 4 Problem frame and path use frame in the model for Barcelona 
Within a problem frame representing these different situations three sections can be distinguished:

- the 'where' section that includes the description of the problem in terms of basic traffic values.

- the 'why' section which includes an interpretation of the causes and severity of the problem. The causes are distributed among the paths that cross the critical section carrying a significant traffic flow. The severity is established from the imbalance between the capacity and the demand in that critical section (excess) according to the demand structure expressed with the paths.

- the 'how' section where the criteria to estimate the level of matching between the real data and the situation represented in the frame are defined.

Two types of frames are considered: generic frames and specific frames. The former are generic characterizations of traffic problems that may appear in different networks with similar physical structures. For instance, the description of an overflowing problem originated on the exit ramp of a road can be described in similar terms for other roads. The difference between them may be the name of the sections and detectors involved and the demand structure. Once the traffic state variables sufficiently match the real time values, the generic frame is instanciated with the specific characteristics of the area and the demand structure is computed for the concrete location of the problem. The specific frames are defined for well known situations where the location, evolution and causes of the problem are known. So, these frames include all the specific elements required to identify a predefined situation.

If some problem frame becames active (i.e. a conflictive situation has been detected) then the Traffic Control unit explores its knowledge base to select control actions to improve the situation. This control knowledge is represented in path-use frames where three sections can also be distinguished:

- the first one includes the definition of a control plan.

- the second one presents the impact on the traffic distribution provoked by the previous plan, i.e. how the selection of paths changes when the plan is active.

- the third one presents a characterization of the traffic situation for which the control plan is appropiate, i.e. which parts of the local network are free and which are congested.

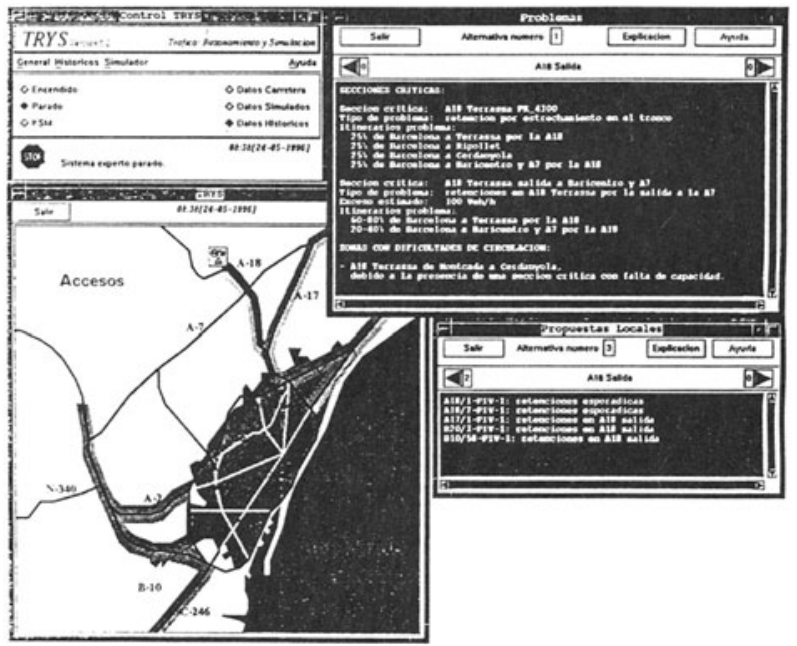

Figure 5 Screen of the TRYS model for Barcelona 
Every preselected signal plan is evaluated through a simulation of its effects on the current traffic situation. This simulation is performed by the 'traffic assignment' method of the Network unit. If this simulated scenario shows a adequate decrease in the flow excess associated to the congestion then the signal plan is delivered to the coordinator else it is rejected. Figure 4 shows a simplified example of a specific problem scenario frame and a path use frame.

The coordinator receives the result of the previous reasoning from every local agent and tries to harmonize the different control plans proposed. In the first stage of the analysis, the coordinator looks for incompatibilities between two or more proposals. Sometimes, the inconsistencies can be solved through a synthesis of the control plans, but in other cases the solution requires the assignment of priorities to the agents involved according to the severity of their problems. At this stage, the coordinator has elaborated a set of global control proposals that may be improved applying strategical criteria from its global view of the traffic network. For instance, it may install a message recommending an interesting alternative path to those drivers entering the traffic network which is not visible by the surrounding local agents.

\section{CONCLUSIONS}

The experience in the application of an open architecture for a real time decision support problem such as the one described in this paper has shown the feasibility and advantages of this approach both for development and maintenance. During the process of development both the generic conceptual model and its contents have been modified several times without relevant losses of time and resources. Also, a good level of user system interaction is available through explanations so it is easy for the user to take decisions based on the system advice.

\section{REFERENCES}

Bholz, C., Dallemagne, G., Klinker, G., Marqués, D. and McDermott, J. (1991) Usable and Reusable Programming Constructs. Knowledge Acquisition, 3, 117-135.

Brachman, R. and Schmolze J. (1985) An overview of the KL-ONE knowledge representation system. Cognitive Science, 9, 171-216.

Bretherton, R.D., Hunt, P.B., Robertson, D.I. and Winton, R.I. (1981) SCOOT: A Traffic Responsive Method of Coordinating Signals. Department of the Environment, Department of Transport, TRRL Report No. LR 1014. Transport and Road Research Laboratory, Crowthorne, UK.

Cuena, J. and Molina, M. (1994) KSM: An Environment for Knowledge Oriented Design of Applications Using Structured Knowledge Architectures. In Applications and Impacts. IFIP'94, Vol 2. K. Brunnstein y E. Raubold (eds.) Elsevier (North-Holland).

Cuena, J., Hernández, J. and Molina M. (1995) Knowledge-Based Models for Adaptive Traffic Management Systems. Transportation Research 3C, 5.

Cuena, J., Hernández, J. and Molina M. (1996) Knowledge Oriented Design of an Application for Real Time Traffic Management: The TRYS System. Proc. of the ECAI'96, Budapest.

Mauro, V. and Di Taranto (1989) UTOPIA. Proc. of the 6th IFAC/IFORS. Conference on Control, Computers and Communications in Transport. Paris.

McIntyre A. (1993) KREST User Manual 2.5. Vrije Universiteit Brussel, AI-Lab. Brussels.

Musen, M.A., Puerta, A.R. and Tu, S.W. (1993) Modeling Tasks with Mechanisms. International Journal of Intelligent Systems, 8. 